Editor's Note: Toolboxes are a new, occasional feature in the Journal designed to briefly highlight a new method or a resource of general use in neuroscience or to critically analyze existing approaches or methods. For more information, see http://www. jneurosci.org/itoa.shtml.

\title{
The National Institutes of Health Blueprint for Neuroscience Research
}

\author{
Robert W. Baughman, ${ }^{1}$ Rebecca Farkas, ${ }^{1}$ Marlene Guzman, ${ }^{2}$ and Michael F. Huerta ${ }^{1}$ \\ ${ }^{1}$ National Institute of Neurological Disorders and Stroke, Bethesda, MD 20892, and ${ }^{2}$ National Institute of Mental Health, Bethesda, MD 20852
}

The National Institutes of Health (NIH) Blueprint for Neuroscience Research is a collaborative effort among the NIH Office of the Director and 16 NIH institutes and centers. ${ }^{a}$ Its aim is to develop research tools, create research resources shared by the entire neuroscience community, train a new generation of cross-disciplinary neuroscientists, and, importantly, to develop a cooperative framework for the institutes and centers to plan and implement their neuroscience research efforts (www.neuroscienceblueprint.nih.gov). The first Blueprint initiatives, launched in 2005, enhanced or expanded access to existing resources like the microarray consortium, the Gene Expression Nervous System Atlas (GENSAT), and the Pediat-

Received Sept. 13, 2006; accepted Sept. 14, 2006.

R.W.B. and M.F.H. are co-chairs, and R.F. and M.G. are co-executive secretaries of the Blueprint Program Coordinating Committee. Story Landis (National Institute of Neurological Disorders and Stroke) and Thomas Insel (National Institute of Mental Health) are the lead Institute Directors for Blueprint. The success of the Blueprint program depends on the contributions of many staff members in the neuroscience community at NIH.

Correspondence should be addressed to Robert W. Baughman at the above address. E-mail:rb175y@nih.gov.

DOI:10.1523/JNEUROSCI.3979-06.2006

Copyright $\odot$ 2006 Society for Neuroscience $\quad$ 0270-6474/06/2610329-03\$15.00/0 ${ }^{a}$ National Center for Complementary and Alternative Medicine (NCCAM), National Center for Research Resources (NCRR), National Eye Institute (NEI), National Institute on Aging (NIA), National Institute on Alcohol Abuse and Alcoholism (NIAAA), National Institute of Biomedical Imaging and Bioengineering (NIBIB), National Institute of Child Health and Human Development (NICHD), National Institute on Drug Abuse (NIDA), National Institute on Deafness and Other Communication Disorders (NIDCD), National Institute of Dental and Craniofacial Research (NIDCR), National Institute of Environmental Health Sciences (NIEHS), National Institute of General Medical Sciences (NIGMS), National Institute of Mental Health (NIMH), National Institute of Neurological Disorders and Stroke (NINDS), National Institute of Nursing Research (NINR), Office of Behavioral and Social Sciences Research (OBSSR). ric MRI Study of Normal Brain Development. Guided by recommendations from the neuroscience research community, the Blueprint developed new initiatives in 2006 for neuroimaging, informatics, core resource centers, behavioral assessment, and specialized training. Future initiatives will address specific scientific themes of interest to all Blueprint institutes and centers: neurodegeneration, neurodevelopment, and plasticity. In this article, we describe the Blueprint resources now available to all neuroscience investigators without regard to their NIH institute or center affiliation, and we summarize funding initiatives for the coming year.

An important goal of the Blueprint is to relieve individual investigators of the time, effort, and financial burden of developing tools on their own, and to achieve this with a negligible impact on funding levels for investigator-initiated neuroscience research. At present, Blueprint activities are funded with just $0.6 \%$ of the total neuroscience research budgets of the 16 participating members, or about $\$ 25$ million per year. This is a very small proportion of all NIH neuroscience funding. The Blueprint has also attracted funds that might not have otherwise been invested in neuroscience. The Blueprint has received an additional $\$ 12$ million per year for four years from the NIH director's discretionary funds. No less important is the NIH commitment of staff for Blueprint activities. More than 125 program directors and other NIH staff have helped launch new initiatives as well as develop programs to coordinate and im- prove access to existing research resources. Many activities in this latter category do not require any Blueprint funding at all.

The list of recent and ongoing Blueprint activities and resources is extensive. Below we summarize some of the major categories of research resources that are now coordinated by the Blueprint program. Currently active FY 2007 Blueprint funding initiatives in neurodegeneration are described in brief under Funding Opportunities.

\section{Blueprint for neuroscience research resources \\ Animal models}

Among the most frequent requests from Blueprint advisory groups are improved access to existing animal models and creation of new models of broad interest. Neuroscience research requires many types of models from primates, to rodents, to invertebrates. The Blueprint website provides links to existing animal model resources (http://www.neuroscienceblueprint. nih.gov/neuroscience_resources/animal_ models.htm). Given the particular importance of mouse models to the study of neurobiology and diseases of the nervous system, the Blueprint is facilitating the sharing and distribution of currently available mouse models and creating new mouse models for future experiments (http://www.neuroscienceblueprint.nih. gov/neuroscience_resources/animal_ models.htm\#rodent). Blueprint supports the sharing of existing mouse models through the NIH Mutant Mouse Regional Resource Centers (MMRRC). A Blueprint 
"repatriation" project will make 220 well characterized transgenic mouse lines, selected by an advisory panel of neuroscientists, available to the neuroscience community. Mouse lines with cell type-specific gene expression from the GENSAT project (see below) (http://www.ncbi. nlm.nih.gov/projects/gensat/) are also being distributed through the MMRRC. In a major boost to increase the tools available for investigating gene function and expression in distinct cell types of the nervous system, Blueprint funded four grants this year to produce and distribute new recombinase-expressing "driver" mouse lines.

\section{Neuroinformatics and computational biology}

Neuroscience research increasingly generates vast amounts of complex and diverse data. Several Blueprint efforts are underway to address the pressing demand for more powerful informatics tools and resources to access and interpret these data. For FY 2006-2007, the Blueprint has launched a pilot effort, the Neuroscience Information Framework (NIF; http:// grants.nih.gov/grants/guide/notice-files/ NOT-DA-05-022.html), to create an efficient means by which laboratory scientists and the public can find resources available for neuroscience research from around the world. The NIF pilot is being developed in conjunction with the Society for Neuroscience Neuroscience Database Gateway, which already provides links to a wide range of neuroscience-related databases. The Blueprint's Neuroimaging Informatics Tools and Resources Clearinghouse (http://www.fbo.gov/servlet/ Solicitation/R/HHS/NIH/NHLBI/NHLBIPB-2006-128) will help scientists make better use of existing neuroimaging informatics tools; many of these resources are greatly underused because they are not user-friendly or easily adaptable to different projects. The web-based Clearinghouse contract will facilitate the dissemination, use, and maintenance of functional magnetic resonance imaging tools. The Blueprint has just established training grants in computational neuroscience to help build a pool of neuroscientists with expertise in computational and mathematical methods. In addition to these new initiatives, the Blueprint Informatics Team is pursuing strategies to link the informatics frameworks that have evolved independently in various labs and research communities over time to boost the value of investments already made and data already collected. This group is exploring ways to encourage data and software sharing, and is providing a collective neuroscience perspective to many informatics efforts emerging across the NIH.

\section{Gene and protein expression}

Resources for determining levels and cellular localization of gene and protein expression are also in high demand. Several such resources are now available through Blueprint. The NIH Neuroscience Microarray Consortium (http://arrayconsortium.tgen.org/np2/home.do), an expansion of a National Institute of Neurological Disorders and Stroke/National Institute of Mental Health resource, offers assistance with experimental design and interpretation of results, and it provides high-quality reagents, services, and training to the neuroscience community on an economical fee-for-service basis. The GENSAT project is mapping gene expression at the cellular level in mouse CNS with a combination of two approaches: a high-throughput in situ hybridization prescreen followed by a high-resolution bacterial artificial chromosome (BAC) transgenic analysis of enhanced green fluorescent protein-reporter gene expression. Data are added to a publicly available online database as they are collected, and all BAC transgenic mice are deposited in the MMRRC mouse repository. With Blueprint support, tissues from visual, auditory, and other sensory pathways are now also being examined in pilot studies.

\section{Cell tissue and DNA}

This is an area where no new Blueprint funds have been invested, but coordination by the Blueprint Repositories Project team has increased the accessibility of a wide range of resources that the individual institutes have developed (http:// www.neuroscienceblueprint.nih.gov/ neuroscience_resources/cell_tissue.htm). These include Human Genetics Repositories and Databases, such as NIH-funded collections of cell lines and DNA with associated clinical data; other cell culture resources, such as NIH-funded cell culture services and stem cell resources; brain banks and other tissue resources, such as $\mathrm{NIH}$-funded repositories with samples of human, nonhuman primate, and rodent nervous system tissue, cerebrospinal fluid, and blood; and cDNA resources, such as NIH-funded collections of human, mouse, zebrafish, and Xenopus cDNA clones, including libraries made from nervous system tissues. This is an extraordinary collection of resources that often were originally collected for a specific pur- pose, but which can be shared by many other research areas.

\section{Imaging tools}

Improved imaging methods and sharing of imaging data and technology are crucial to current research in neuroscience. The Blueprint is supporting the development of new methods to visualize neural activity with much higher spatial and temporal resolution than is currently possible. The Blueprint is also supporting the expansion of the NIH Pediatric MRI Study of Normal Brain Development to include diffusion tensor imaging (DTI) and the development of new tools for analyzing DTI data. The data collected from this project will enable the study of normal brain development, provide reliable control data for studies of childhood disorders and diseases that affect the brain, and may aid in the development of new diagnostic tools.

\section{Equipment and infrastructure}

The Blueprint has funded four Neuroscience Blueprint Interdisciplinary Center core grants (http:/grants.nih.gov/grants/ guide/rfa-files/RFA-NS-06-003.html) to provide resources for a range of local, regional, and national interdisciplinary core facilities. In addition, several other programs are available that support shared core resources, equipment, and infrastructure (http://www.neuroscienceblue print.nih.gov/neuroscience_resources/ equipment.htm).

\section{Neurological and behavioral assessment} Many neurological and behavioral studies collect data on cognitive, sensory, and/or motor aspects of neural function, but there is little uniformity among the measures used. It is difficult to draw comparisons or compile data across studies that use unique assessment test batteries. To establish standard assessment measures that address this weakness, the Blueprint has funded a contractor to develop an NIH Toolbox for Assessment of Neurological and Behavioral Function (http:// grants.nih.gov/grants/guide/notice-files/ NOT-AG-06-008.html). This initiative will develop a set of neurological and behavioral measurements appropriate for a variety of project types, particularly longitudinal epidemiological studies and prevention or intervention trials.

\section{Training}

The advance of research in neuroscience increasingly requires interdisciplinary training that is not readily supported under the programs of the individual NIH institutes and centers. The Blueprint, 
therefore, has targeted specific areas for interdisciplinary training programs. In FY 2006, the Blueprint funded initiatives for Training in Translational Research in Neurobiology of Disease (T32 Institutional National Service Award), Training in Neuroimaging (T90 Interdisciplinary Research Training Award), Training in Computational Neuroscience (T90 Interdisciplinary Research Training Award), and Course Development in the Neurobiology of Disease (R25 Education Projects). In fiscal year (FY) 2007, Blueprint will support initiatives for Interdisciplinary Individual Postdoctoral Fellows for Training in Neurodegeneration Research (F32 Postdoctoral Individual National Research Award) and Short-Term Interdisciplinary Career Enhancement Awards for Neurodegeneration Research (K18 Career Enhancement Award) (see FY 2007 links below).

The neuroscience research resources and tools listed here are just a portion of those that are now accessible through the Blueprint website. Investigators should visit this site to see whether there are other opportunities for enhancing their individual research efforts.

\section{Blueprint for neuroscience research funding opportunities}

In its first two years of operation, the Blueprint sponsored 14 funding initiatives (http://neuroscienceblueprint.nih.gov/ blueprint_funding/funding_archive. htm). Many of these initiatives were co- funded by one or more individual institutes, often at a substantial level. Starting in FY 2007, the Blueprint initiatives yearby-year will focus on scientific thematic areas. The themes will be neurodegeneration, neurodevelopment, and plasticity in FY 2007, 2008, and 2009, respectively.

To provide guidance for the FY2007 neurodegeneration effort, a public Request for Information (RFI) for initiative concepts was released in the NIH Guide, and a planning workshop was held in March 2006. The workshop brought together approximately 30 scientists from a broad range of disciplines and perspectives to identify research tools, resources, and training activities that could accelerate progress in neurodegeneration research (http://www.neuroscienceblueprint.nih.gov/ blueprint_basics/200603_workshop_ report.htm). Based on the recommendations of the meeting, feedback from the RFI, and the available financial resources, a series of four initiatives was released in September 2006. These include Therapeutics Delivery for Neurodegenerative Diseases (R21 Exploratory/Developmental Grants; http:/grants.nih.gov/grants/ guide/rfa-files/RFA-EY-07-001.html), Biomarkers for Neurodegeneration (R21 Exploratory/Developmental Grants; http:/grants.nih.gov/grants/guide/rfafiles/RFA-NS-07-004.html), Interdisciplinary Individual Postdoctoral Fellowships for Training in Neurodegeneration Research (F32 Postdoctoral Individual National Research Service Award; http:// grants.nih.gov/grants/guide/rfa-files/ RFA-AG-07-004.html), and Short-Term Interdisciplinary Career Enhancement Awards for Neurodegeneration Research (K18 Career Enhancement Award; http:// grants.nih.gov/grants/guide/rfa-files/ RFA-DC-07-005.html). See the NIH Guide for the full announcements.

To solicit similar guidance from the field for the neurodevelopment initiatives in FY 2008, a public request for information was released in the NIH Guide (http:/grants.nih.gov/grants/guide/noticefiles/NOT-MH-06-114.html), and a planning workshop for initiative concepts for neurodevelopment will be held in November 2006. Note that the closing date for responses to the Neurodevelopment RFI is October 31, 2006.

\section{Summary}

In summary, the NIH Blueprint for Neuroscience Research has become the central focus for coordination of NIH support for neuroscience research. With a very modest investment, it has created a framework for sharing resources and providing training opportunities to leverage the strengths of the participating institutes and centers. All NIH neuroscience investigators, independent of their source of funding, now have access to tools, resources and training that individual institutes could not provide. The Blueprint will continue to serve this role with input and guidance from the field. The Blueprint welcomes public comments via email (blueprint@mail.nih.gov). 